OPEN ACCESS

Edited by:

Richard Porter,

University of Otago,

New Zealand

Reviewed by:

Jennifer Jordan,

University of Otago,

Christchurch, New Zealand

Lee Kannis-Dymand,

University of the Sunshine

Coast, Australia

*Correspondence:

Adrian Wells

Adrian.wells@manchester.ac.uk

Specialty section:

This article was submitted to Mood and Anxiety Disorders,

a section of the journal

Frontiers in Psychiatry

Received: 13 March 2020 Accepted: 05 June 2020

Published: 30 June 2020

Citation:

Wells $A$, Reeves $D$, Heal $C$, Fisher $P$, Davies L, Heagerty A, Doherty $P$ and Capobianco L (2020) Establishing the

Feasibility of Group Metacognitive Therapy for Anxiety and Depression in Cardiac Rehabilitation: A Single-Blind Randomized Pilot Study.

Front. Psychiatry 11:582. doi: 10.3389/fpsyt.2020.00582

\section{Establishing the Feasibility of Group Metacognitive Therapy for Anxiety and Depression in Cardiac Rehabilitation: A Single-Blind Randomized Pilot Study}

\author{
Adrian Wells ${ }^{1,2 *}$, David Reeves ${ }^{3}$, Calvin Heal ${ }^{3}$, Peter Fisher ${ }^{4,5}$, Linda Davies ${ }^{6}$, \\ Anthony Heagerty ${ }^{7,8}$, Patrick Doherty ${ }^{9}$ and Lora Capobianco ${ }^{2}$
}

1 Faculty of Biology, Medicine and Health, School of Psychological Sciences, The University of Manchester, Manchester, United Kingdom, ${ }^{2}$ Research and Innovation, Greater Manchester Mental Health NHS Foundation Trust, Manchester, United Kingdom, ${ }^{3}$ NIHR School for Primary Care Research, Manchester Academic Health Science Centre, The University of Manchester, Manchester, United Kingdom, ${ }^{4}$ Institute of Psychology, Health and Society, University of Liverpool, Liverpool, United Kingdom, ${ }^{5}$ Liverpool Clinical Health, The Royal Liverpool and Broadgreen University Hospital NHS Trust, Liverpool, United Kingdom, ${ }^{6}$ Centre for Health Economics, Division of Population Health. Health Services Research and Primary Care, Faculty of Biology Medicine and Health, School of Health Sciences, The University of Manchester, Manchester, United Kingdom, ${ }^{7}$ Core Technology Facility, The University of Manchester School of Medical Sciences, Manchester, United Kingdom,

${ }^{8}$ Manchester University NHS Foundation Trust, Manchester Royal Infirmary, Manchester, United Kingdom, ${ }^{9}$ Department of Health Sciences, University of York, York, United Kingdom

Background: Anxiety and depression are common in cardiac rehabilitation (CR) patients. However, CR programs which incorporate psychological techniques achieve modest reductions in emotional distress. More efficacious interventions that can be easily integrated within services are required. A promising alternative to current psychological interventions is metacognitive therapy (MCT). The aim was to evaluate the acceptability and feasibility of delivering Group-MCT to CR patients experiencing symptoms of anxiety and depression.

Method and Results: Fifty-two CR patients with elevated anxiety and/or depression were recruited to a single-blind randomized feasibility trial across three UK National Health Service Trusts and randomized to usual CR or usual CR plus six weekly sessions of group-MCT. Acceptability and feasibility of adding group-MCT to CR was based on recruitment rates, withdrawal, and drop-out by the primary end-point of 4 months; number of MCT and CR sessions attended; completion of follow-up questionnaires; and ability of the outcome measures to discriminate between patients. The study was also used to re-estimate the required sample size for a full-scale trial. We also examined the extent by which non-specialists adhered to the Group-MCT protocol. Group-MCT was found to be feasible and acceptable for CR patients with anxiety and depression. Recruitment and retention of participants was high, and attendance rates at CR were similar for both groups. 


\section{Conclusion: The results suggest the addition of MCT to CR did not have a negative impact on retention and support a full-scale trial of Group-MCT for cardiac patients.}

Keywords: cardiac rehabilitation, anxiety, depression, mental health, metacognitive therapy

\section{INTRODUCTION}

Heart and circulatory disease is associated with approximately 170,000 deaths each year in the UK (1). However, survival rates are improving, and approximately 7.4 million people are living with heart and circulatory disease in the UK (1). Rehabilitation following a cardiac event is vital for improving health outcomes, quality of life, and survival. The UK Department of Health, National Institute for Health and Care Excellence (NICE) guidelines (CG172, CG94, and NG106), and the British Association for Cardiovascular Prevention and Rehabilitation (BACPR) recommend that cardiac rehabilitation (CR) programs are offered to all eligible patients (2-4).

Modern CR programs are comprised of exercise training, psychosocial support, risk factor management, and education underpinned by a behavior change approach (2). CR is costeffective $(5,6)$, reduces mortality and morbidity $(5,7)$, and increases quality of life $(5,8)$. However, improvement of anxiety and depression in cardiac rehabilitation is limited. Anxiety and depression are highly prevalent among CR patients, with $27.6 \%$ of CR patients experiencing clinically significant anxiety and $19 \%$ experiencing clinically significant depression (9). Furthermore, elevated anxiety and depression have been associated with decreased medication adherence, decreased CR attendance (10), decreased quality of life, increased risk of reoccurrence of cardiac related events, and a higher rate of mortality, leading to greater service use and increased NHS costs $(11,12)$.

Combined with the low rates of provision of psychological treatment, those treatments that have been evaluated appear to have limited efficacy. Current interventions for anxiety and depression for patients with a physical illness often focus on cognitive behavioral therapy (CBT). A recent Cochrane systematic review and meta-analysis evaluated psychological interventions in coronary heart disease in comparison to treatment as usual, and included 35 studies. Common components of psychological interventions included relaxation techniques, self-awareness and self-monitoring, emotional support/client-led discussion, and cognitive challenging/ cognitive restructuring. At post-treatment interventions were associated with a between group effect size of 0.27 (Cohen's d) for symptoms of depression, with similar results for symptoms of anxiety (Cohen's $\mathrm{d}=0.24)(13)$. This is in line with previous studies that highlight the limited efficacy of psychological interventions in cardiac patients $(14,15)$. It is evident that more effective interventions that can be easily integrated within services are required.

Metacognitive therapy (MCT) (16), is a transdiagnostic therapy found to be efficacious in mental health settings [see (17)]. MCT might be well suited to the psychological needs of cardiac patients because it does not depend on challenging the validity of negative thoughts and beliefs which can be realistic in people suffering from chronic and potentially lifethreatening conditions. MCT is based on the self-regulatory executive function model (S-REF model) $(18,19)$ of psychological disorders. In the S-REF model, anxiety, depression, and adjustment difficulties are maintained by the activation of a maladaptive thinking style called the cognitive attentional syndrome (CAS). The CAS is characterized by worrying, rumination, inflexible attention to threat, and maladaptive coping strategies. The CAS is driven by underlying metacognitive beliefs, which can be differentiated into positive and negative subtypes. Positive metacognitive beliefs concern the usefulness of worry (e.g. "worrying helps to detect problems before it is too late") while negative metacognitive beliefs concern uncontrollability (e.g. "I cannot stop worrying about the future") and dangerousness of worry (e.g. "worrying will cause a heart attack").

Normann and Morina (17) conducted a systematic review and meta-analysis of the efficacy of metacognitive therapy for anxiety and depression and found that MCT demonstrated a large between group effect size at post treatment (Hedges' $\mathrm{g}=$ 2.06) when compared to a waitlist control condition. When MCT was compared to cognitive and behavioral interventions MCT also demonstrated large effect sizes favoring MCT at post treatment (Hedges' $\mathrm{g}=0.69$ ). MCT has also shown promising results when delivered using a group format in mental health settings. To date there have been nine studies evaluating GroupMCT across various disorders including obsessive compulsive disorder $(20,21)$, generalized anxiety disorder (22-24), depression $(25,26)$, and in mixed disorder groups $(27,28)$. No studies to date have compared Group-MCT with individual MCT, but a preliminary comparison of Group-MCT with group mindfulness based stress reduction in mixed anxiety and depression groups found that both were feasible and acceptable, with large within-subject effects (27). In physical health conditions MCT has primarily been evaluated in cancer, with promising pilot results (29-31).

While positive outcomes for MCT within mental health settings are rapidly gaining support, accelerated research efforts are needed in the application to treating psychological distress symptoms in physical health. As such the aims of the current study were to evaluate the acceptability and feasibility of delivering group metacognitive therapy within cardiac rehabilitation services, the present study was intended to serve as a feasibility for a subsequent definitive trial [trial protocol: (32)]. Our research questions were: (1) What are the recruitment and retention rates? (2) Do therapists adhere to the treatment protocol? (3) What proportion of the treatment (i.e. CR and Group-MCT) is completed by patients? (4) What is the rate of 
return of measures and the variability in scores for primary and secondary outcomes? (5) Are there any adverse events or serious adverse events in the intervention group? (6) What is the sample size required for a full-scale trial?

\section{METHODS}

\section{Design}

The PATHWAY Group-MCT pilot feasibility study is a multicenter randomized controlled trial with 4- and 12month follow-up comparing Group-MCT plus usual cardiac rehabilitation (intervention) versus usual cardiac rehabilitation (control). The study served as an internal pilot to the full-scale RCT (32). The study was approved by the National Research Ethics Service of the UK's National Health Service (ref 14/NW/ 0163) and registered with a clinical trial data base (ISRCTN reference: ISRCTN74643496).

Patients referred to CR programs in the UK are routinely sent a National Audit of Cardiac Rehabilitation assessment pack (9), which includes the Hospital Anxiety and Depression Scale (HADS) (33). Participants were recruited from three NHS cardiac rehabilitation services in the North West of England (University Hospital of South Manchester NHS Foundation Trust, Central Manchester University Hospitals NHS Foundation Trust, and East Cheshire NHS Trust). At the initial assessment appointment, CR staff reviewed each patient's medical notes for inclusion and exclusion criteria, initially screening for those who scored eight or above on either the HADS anxiety and or depression subscale, indicative of at least "mild" symptomatology. In addition, participants had to meet the Department of Health and/or British Association for Cardiac Prevention and Rehabilitation CR eligibility criteria, be aged 18 years or older, and have a competent level of English language (able to read, understand, and complete questionnaires in English). Patients who were eligible for the trial were invited to take part. Inclusion and exclusion criteria are provided in further detail in Wells et al. (32). Eligible participants were contacted by research assistants and participants were provided with further information on the trial and had the opportunity to ask questions about participation in the study. Participants were consented face-to-face with a research assistant either at the patient's home or at the site of their CR. Research assistants taking consent, the chief investigator and trial statisticians were blind to each patient's treatment allocation. Data monitoring, quality, and handling were undertaken by the Manchester Clinical Trials unit and project oversight was by an independent trial steering committee. The trial has been reported in line with CONSORT statement for the reporting of pilot and feasibility randomized controlled trials (34).

\section{Patient and Public Involvement}

Patient and public involvement (PPI) has been extensively involved throughout the course of the study. PPI service users were first involved in grant development and commissioning of the research project. After which, PPI service users were subsequently involved in participant recruitment and ensuring that the number and length of outcome measures selected did not overburden participants, as well as aiding in recruitment and retention of participants. Specifically, PPI service users aided in co-producing a newsletter sent out to patients following 4-month follow-up to remind patients of upcoming follow-up questionnaires and updating participants about the study. PPI service users have also aided in co-producing a dissemination plan and aiding in dissemination of research findings.

\section{Participants}

The Consort diagram indicating patient flow is depicted in Figure 1. Fifty-two eligible patients were randomized to either usual cardiac rehabilitation (control) or to usual cardiac rehabilitation plus group metacognitive therapy (intervention).

\section{Randomization and Sample Size}

Following informed consent, patients were randomly allocated to a trial condition in a 1:1 ratio using a minimization algorithm that incorporated a random component, in order to maximize balance between the two arms on sex distribution, HADS anxiety and depression scores, and hospital site. Table 1 provides a summary of randomization variables by group. Randomization was conducted via a telephone link to the Manchester University Clinical Trial Unit (Manchester CTU).

The target sample size was originally 50 patients ( 25 per arm), determined as sufficient to evaluate recruitment and retention rates for a full-scale trial as well as rates of completion of the intervention. This sample is also adequate for estimation of variability in outcome measures for which samples of 40 are considered sufficient (35). However, as a consequence of parallel recruitment across sites, 52 patients had consented by the end of the recruitment period and were included in the sample. Patterns of participant sex and HADS subscale scores resulted in 23 of these being allocated by computer to the intervention group and 29 to the controls. Sample characteristics are in Table 2.

\section{Treatment}

\section{Treatment as Usual (TAU; Control)}

Treatment as usual was the CR program delivered at each study site in hospital and community settings. CR programs are comprised of an exercise component and an educational component. Both components are provided in group sessions over 8 to 10 weeks, of 45 to $60 \mathrm{~min}$. The therapist-to-patient ratio in TAU exercise sessions is 1:5 for low- and moderate-risk patients and 1:3 for high-risk patients. In addition to exercise classes, educational seminars are delivered that focus on a variety of topics including lifestyle and medical risk factor management. TAU educational sessions varied by site and the extent of psychological components also varied. While all sites delivered sessions on relaxation which focused on breathing techniques and progressive muscle relaxation, the content of sessions on stress management differed. For example, two sites incorporated cognitive therapy methods (i.e. challenging negative thoughts, worry decision tree), while one site delivered psychoeducational information on stress. In addition, one site offered a 4-week stress management course as part of CR which included 


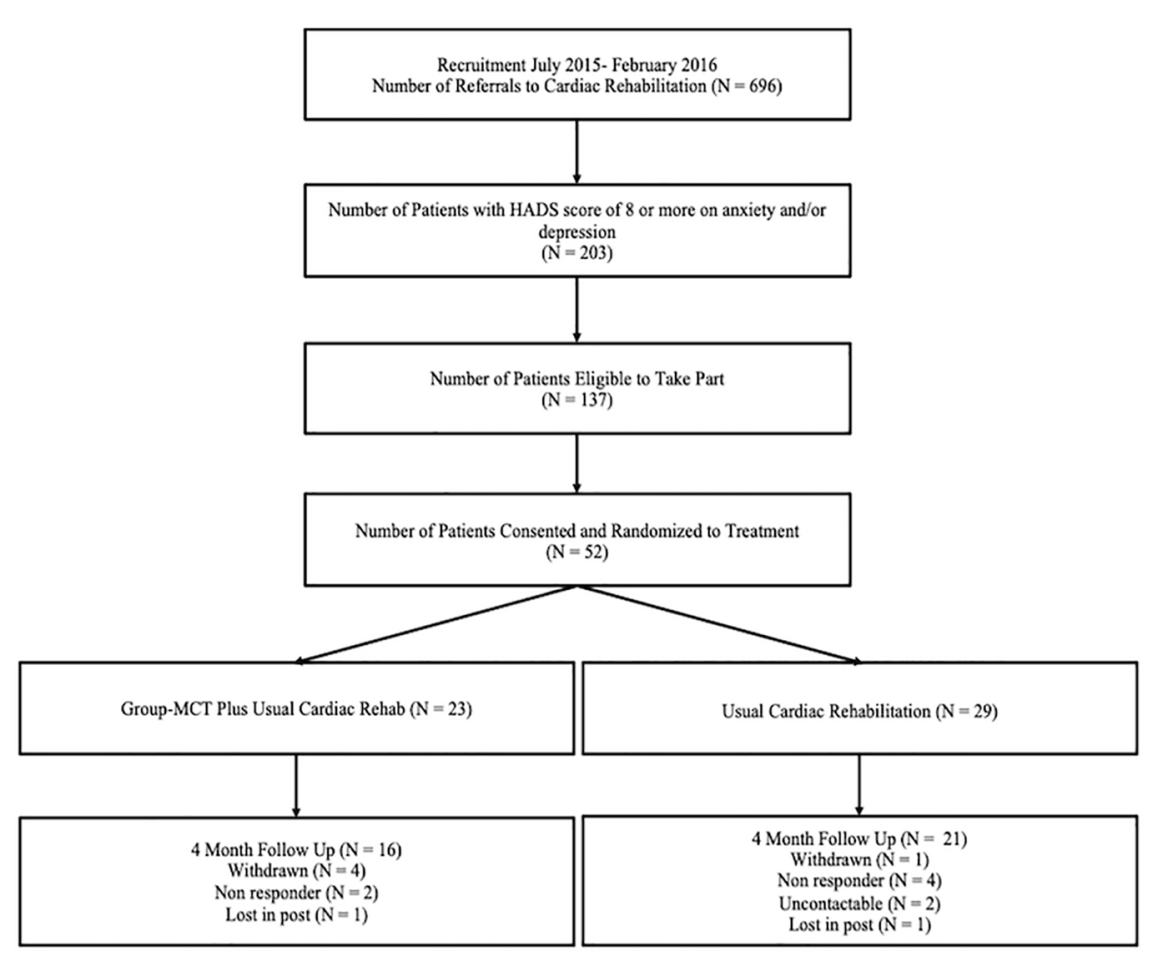

FIGURE 1 | CONSORT flow diagram of patient recruitment.

TABLE 1 | Sample characteristics by Randomization Variable per Group.

\begin{tabular}{|c|c|c|c|c|c|c|}
\hline \multirow[t]{2}{*}{ Arm } & \multicolumn{2}{|c|}{$\begin{array}{c}\text { HADS Anxiety } \\
\geq 8\end{array}$} & \multicolumn{2}{|c|}{$\begin{array}{l}\text { HADS Depres- } \\
\text { sion } \geq 8\end{array}$} & \multicolumn{2}{|c|}{$\begin{array}{c}\text { HADS Anxiety anc } \\
\text { Depression } \geq 8\end{array}$} \\
\hline & Male & Female & Male & Female & Male & Female \\
\hline TAU & 6 & 3 & 2 & 2 & 9 & 7 \\
\hline TAU + Group-MCT & 5 & 2 & 2 & 0 & 8 & 6 \\
\hline
\end{tabular}

TAU, treatment as usual; MCT, metacognitive therapy; HADS, Hospital Anxiety and Depression Scale.

generating and sharing a case formulation based on Greenberger and Padesky (36), mindfulness techniques, and individual counseling with an occupational therapist.

\section{Group-MCT Plus Treatment as Usual (Group-MCT; Intervention)}

Group-MCT was delivered in addition to usual CR. Group-MCT was most often delivered on a different day to participants' CR sessions, and only four intervention patients attended CR and Group-MCT on the same day. Group-MCT was delivered in six sessions of 60- to 90-min duration and followed a treatment manual (37). Sessions focused on deriving a case formulation and socialization, practicing the Spatial Attentional Control Exercise (SpACE), detached mindfulness (DM), worry and rumination postponement, challenging metacognitive beliefs concerning the uncontrollability and danger of worrying and rumination, and developing a "helpful behaviors" plan. The manual contains techniques that help patients to change the type of relationship that they have with their negative thoughts, allowing them to step back and not engage in worry and/or rumination. One of the techniques, SpACE, is a brief auditory exercise consisting of instructions to allocate attention in different ways to discover control over thinking while inhibiting responses to spontaneous negative thoughts and prolonged attention capture by distractions. The technique is designed to increase mental flexibility so that negative thoughts and memories can be de-coupled from repetitive negative thinking. DM is a technique used in Group-MCT in which the patient learns to relate to thoughts and disengage from unhelpful coping strategies. Patients were asked to practice SpACE twice a day for homework and to practice applying DM to negative thoughts. SpACE practice was recorded in a personal diary and homework was reviewed at the start of each session with discussions around successes and difficulties experienced. However, data on practice were captured for analysis purposes.

\section{Therapists}

Group-MCT was delivered by CR staff who had received a basic training in implementing the treatment manual. There were seven therapists, two therapists per site except one site where three therapists were trained. Therapists included occupational therapists, physical therapists, and CR nurses. Therapists completed a 2-day workshop delivered by the developer of MCT (AW). Training included didactic teaching, role play, discussion, and studying the treatment manual. In addition, therapists delivered the intervention to a pilot group of volunteers along with an additional 1-day workshop, which focused on enhancing the initial skills. Therapists received ongoing supervision on an occasional basis, while delivering the intervention. 
TABLE 2 | Baseline Demographic and Clinical Characteristics.

\begin{tabular}{|c|c|}
\hline Demographic factors & $\begin{array}{c}\text { Entire sample ( } \mathrm{N}=52 \\
\mathrm{n}(\%)\end{array}$ \\
\hline \multicolumn{2}{|l|}{ Sex } \\
\hline Male & 33 (63.5\%) \\
\hline Female & 19 (36.5\%) \\
\hline \multicolumn{2}{|l|}{ Ethnicity } \\
\hline Any white & $43(82.7 \%)$ \\
\hline All other categories & $8(15.4 \%)$ \\
\hline \multicolumn{2}{|l|}{ Psychological therapies for anxiety or depression } \\
\hline In the past & 18 (34.6\%) \\
\hline Never & 34 (65.4\%) \\
\hline Age & $58(9.6)$ \\
\hline \multicolumn{2}{|l|}{ Employment: } \\
\hline Economically active & $18(34.6 \%)$ \\
\hline Unemployed & $8(15.4 \%)$ \\
\hline Retired & $16(30.8 \%)$ \\
\hline All other & $10(19.2 \%)$ \\
\hline \multicolumn{2}{|l|}{ Educational qualification } \\
\hline None & 16 (30.8\%) \\
\hline School/vocational & $22(42.3 \%)$ \\
\hline Diploma/degree & 14 (26.9\%) \\
\hline \multicolumn{2}{|l|}{ Civil status: } \\
\hline In relationship & 26 (50.0\%) \\
\hline Separated & 18 (34.6\%) \\
\hline Single & $8(15.4 \%)$ \\
\hline \multicolumn{2}{|l|}{ Smoking status } \\
\hline Never smoked & 16 (30.8\%) \\
\hline Ex-smoker & $31(59.6 \%)$ \\
\hline Current smoker & $5(9.6 \%)$ \\
\hline \multicolumn{2}{|l|}{ Alcohol units per month } \\
\hline None & 21 (40.4\%) \\
\hline 1 to 19 & 17 (32.7\%) \\
\hline 20 to 49 & $7(13.5 \%)$ \\
\hline 50 or more & 7 (13.5\%) \\
\hline \multicolumn{2}{|l|}{ Age at first cardiovascular event } \\
\hline Under 45 years & $5(9.6 \%)$ \\
\hline 45 to 54 years & $21(40.4 \%)$ \\
\hline 55 years and older & 25 (48.1\%) \\
\hline \multicolumn{2}{|l|}{ Number of cardiac events } \\
\hline None & 38 (73.1\%) \\
\hline 1 & $7(13.5 \%)$ \\
\hline 2 or more & $7(13.5 \%)$ \\
\hline \multicolumn{2}{|l|}{ BMl } \\
\hline Underweight/normal & 14 (26.9\%) \\
\hline Overweight & 21 (40.4\%) \\
\hline Obese & $15(28.9 \%)$ \\
\hline Number of comorbidities [mean (SD)] & $5.1(2.1)$ \\
\hline Outcomes & Mean (SD) \\
\hline HADS total & $17.5(5.7)$ \\
\hline HADS anxiety & $10.1(3.6)$ \\
\hline HADS depression & $7.4(3.5)$ \\
\hline Impact of Event Scale-Revised (IESR) & $32.6(19.2)$ \\
\hline Metacognitions Questionnaire 30 (MCQ-30) & $62.3(15.1)$ \\
\hline EQ-5D-5L Utility & $0.60(0.3)$ \\
\hline EQ-5D VAS & $61.5(18.5)$ \\
\hline CAS-1R & $394.1(185.2)$ \\
\hline
\end{tabular}

\section{Measures}

Outcome Measures

Hospital Anxiety and Depression Scale (HADS)

The Hospital Anxiety and Depression Scale [HADS; Zigmond and Snaith (33)] was specified as the primary outcome for the pilot trial and subsequent main RCT and is a 14-item measure that assesses symptoms of anxiety and depression, where items are scored on a 4-point Likert scale (0 to 3 ). The scale generates two subscale scores (anxiety and depression) and a total score. The total score was the pre-specified primary outcome measure. The HADS total score has demonstrated good internal consistency with Cronbach's alpha for the total score in cardiac patients ranging from 0.85 to $0.89(38,39)$.

\section{Impact of Events Scale-Revised (IES-R)}

The Impact of Event Scale-Revised [IES-R; Weiss and Marmar (40)] is a 22-item scale assessing symptoms of post-traumatic stress disorder across three subscales, intrusions, avoidance, and hyperarousal, plus a single total score. Each item is rated from 0 (not at all) to 4 (extremely) based on how distressing the item has been in the past week with respect to their cardiac event. The scale has shown high internal consistency across all three subscales: intrusion Cronbach's alpha $=0.87$ to 0.94 ; avoidance Cronbach's alpha $=9.84$ to 0.87 ; hyperarousal Cronbach's alpha $=0.79$ to 0.91 (31-33); total Cronbach's alpha $=0.95$ (41). The subscales also demonstrate moderate to high inter-correlations ( $\mathrm{rs}=0.52-0.87)(41$, 42). We pre-specified the total score as the outcome for this study.

\section{Metacognitions Questionnaire-30 (MCQ-30)}

The Metacognition Questionnaire-30 [MCQ-30; Wells and Cartwright-Hatton (43)] is a 30 item measure that assesses metacognitive beliefs across five subscales: (1) positive metacognitive beliefs, (2) negative metacognitive beliefs regarding uncontrollability and danger, (3) cognitive confidence, (4) cognitive self-consciousness, and (5) need to control thoughts. Items are scored on a four-point Likert scale (do not agree to agree very much), whereby higher scores indicate greater maladaptive metacognitive beliefs. A total score is also derived and was used as the outcome measure for this study. The MCQ-30 possesses good internal consistency (Cronbach's alphas 0.72 to 0.93 for individual subscales) $(43,44)$.

\section{Cognitive Attentional Syndrome-1 Revised (CAS-1R)}

The Cognitive Attentional Syndrome-1 Revised [CAS1-R; Wells (45)] is a 10-item measure that assesses different aspects of the CAS across three subscales: coping strategies, positive metacognitive beliefs, and negative metacognitive beliefs. The measure was modified from the original CAS-1 (16) for use in PATHWAY. Items are rated on an 11-point Likert scale ranging from 0 (none of the time/not at all true) to 100 (all of the time/completely certain this is true), whereby higher scores indicate greater use of maladaptive coping strategies or maladaptive metacognitive beliefs. The psychometric properties of the CAS-1R have been evaluated in cardiac patients (46). The measure demonstrates acceptable internal consistency for two subscales: coping strategies (Cronbach's alpha $=0.88)$ and negative metacognitive beliefs (Cronbach's alpha $=0.65)$; however, internal consistency was low for the positive metacognitive beliefs subscale (Cronbach's alpha $=$ 0.58). The scale demonstrates good construct validity and is a significant predictor of anxiety and depression in cardiac patients.

\section{EQ-5D-5L}

The EQ-5D-5L [EuroQol (47)] describes and values health across five dimensions: mobility, self-care, usual activities, pain/ discomfort, and anxiety/depression (48). Response items range 
from no problem to extreme problem. The EQ-5D-5L can be calculated as a total score and also converted to a utility score. In addition, participants rate their overall health using a visual analog scale (VAS), which ranges from 0 (worst health imaginable) to 100 (best health imaginable).

\section{Statistical Analysis}

Statistical analysis was principally descriptive. We assessed the acceptability of adding Group-MCT to usual CR in terms of rates of recruitment into the study (number agreeing to participate out of those approached, and number recruited per month), withdrawal or drop-out by the primary endpoint of 4 months (attrition rate), and numbers of MCT and CR sessions attended.

The feasibility of conducting a full trial was assessed with regard to completion of follow-up questionnaires (proportions of missing values, both overall and within trial arms), ability of the outcome measures to discriminate between patients (range of scores; floor or ceiling effects), and re-estimation of the required sample size based on the findings of this study (number of recruited patients required to detect an effect size of 0.4 on HADS total score at $80 \%$ power, controlling for baseline scores and allowing for attrition and clustering of patients within therapy groups). We examined therapist level of adherence to the treatment protocol since these individuals were non-mental health specialists without prior experience of delivering psychological treatments. An adherence checklist was used for this purpose, whereby therapists indicated the aspects of the protocol implemented in each session, if they had been missed and if so why. For example, in the first session adherence items included completing the case formulation, socializing patients to the model, practicing SpACE, and assigning homework. Consistently evaluated elements in each session included reviewing and assigning homework and practicing SpACE. A total adherence score was created for each session by summing the total number of elements completed in session.

\section{RESULTS}

\section{Participants}

52 participants (19 female, 33 male) took part in the study. Patients had a mean age of 58.10 (SD $=9.61$, range: $38-79$ ). Participants ethnic origin was primarily white (82.7\%); however, eight participants identified as the following ethnic origins: Asian Bangladeshi $(\mathrm{n}=1)$, Asian Indian $(\mathrm{n}=1)$, Asian Pakistani $(\mathrm{n}=$ $1)$, Any other Asian background $(n=1)$, Black Caribbean $(n=3)$, and any other Black background $(n=1)$. Patients had a range of heart conditions including: acute coronary syndrome $(\mathrm{n}=31)$, revascularization $(n=26)$, heart failure $(n=9)$, angina $(n=3)$, implantation of cardioverter defibrillator $(n=1)$, heart valve repair/replacement $(n=8)$, and adult congenital heart disease $(\mathrm{n}=2)$. At initial assessment, the mean anxiety score was 10.02 $(\mathrm{SD}=3.27)$, and the mean depression score was $8.48(\mathrm{SD}=3.13)$. 30 patients met criteria for both anxiety and depression, 16 patients met criteria for anxiety only, and 6 met criteria for depression only.

\section{Acceptability}

Patients and therapists were asked about the acceptability of the treatment in a separate qualitative study that will be reported in detail elsewhere. The qualitative reports suggested that both patients and therapists found the treatment to be logical and appropriate for the problems being addressed.

\section{Feasibility Assessment Recruitment}

Participant recruitment took place over 8 months across three CR services in the North West of England. During this time 696 patients were referred to CR programs, of which 203 (29.2\%) had a score of 8 or more on either or both HADS subscales. 137 patients met the study eligibility criteria, and 119 of these were provided with further information on the study. Of the 119, 14 started CR before they could be offered the trial (making them ineligible), 21 were missed (i.e. not screened for eligibility), while another 32 declined to participate. The remaining 52 (38\% of those eligible to take part) were consented and randomized into the study. As such, over 8 months the rate of recruitment was approximately 6.5 patients consented and randomized per month.

\section{Outcome Measures}

To maintain the masking of the trial statistician (necessary for the inclusion of this data in the definitive RCT) the analyses of the outcome measures in the current study has been conducted on the full sample only. There was no missing data on any outcome measure at baseline. Table 3 provides a summary of the

TABLE 3 | Descriptive Statistics on Outcome Measures at Baseline.

\begin{tabular}{|c|c|c|c|c|c|c|}
\hline Outcome measure & $\begin{array}{l}\text { Sample } \\
\text { Size }\end{array}$ & $\begin{array}{c}\% \\
\text { Missing }\end{array}$ & $\begin{array}{l}\text { Median (inter- } \\
\text { quartile range) }\end{array}$ & $\begin{array}{l}\text { Minimum and maximum } \\
\text { observed scores }\end{array}$ & $\begin{array}{l}\text { Minimum possible score } \\
\text { (\% scoring minimum) }\end{array}$ & $\begin{array}{l}\text { Maximum possible score } \\
\text { (\% scoring maximum) }\end{array}$ \\
\hline $\begin{array}{l}\text { Hospital Anxiety and Depression } \\
\text { Scale (total score) }\end{array}$ & 52 & 0 & $17(13.5-22)$ & 1,28 & $0(0 \%)$ & $42(0 \%)$ \\
\hline $\begin{array}{l}\text { Impact of Event Scale-Revised } \\
\text { (total) }\end{array}$ & 52 & 0 & $30.5(18-45)$ & 1,85 & $0(0 \%)$ & $88(0 \%)$ \\
\hline $\begin{array}{l}\text { Metacognitions Questionnaire } 30 \\
\text { (total) }\end{array}$ & 52 & 0 & $60(52.5-72.6)$ & 32,97 & $30(0 \%)$ & $120(0 \%)$ \\
\hline EQ-5D-5L (total) & 52 & 0 & $10(8-14)$ & 5,19 & $5(7.7)$ & $25(0 \%)$ \\
\hline EQ-5D-5L utility score & 52 & 0 & $0.66(0.45-0.80)$ & $-0.065,1$ & $-0.594(0 \%)$ & $1(7.7 \%)$ \\
\hline
\end{tabular}



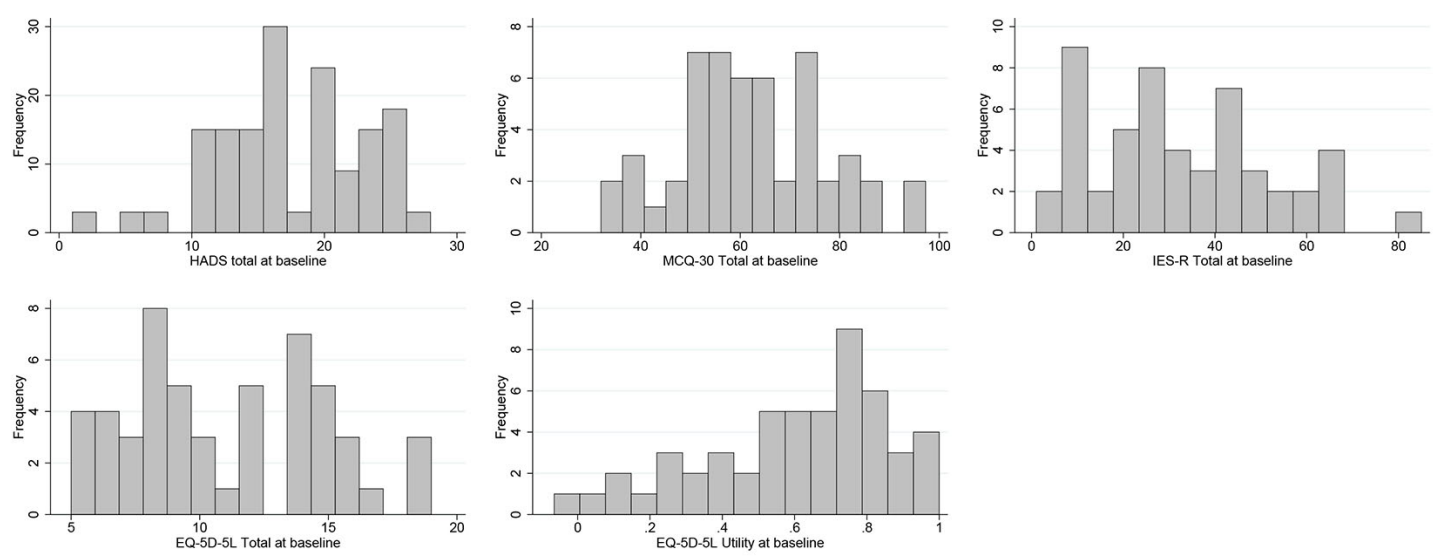

FIGURE 2 | Outcome measure histograms.

descriptive statistics for each outcome measure and Figure 2 provides a histogram for each. All questionnaires demonstrated a good range of observed scores, covering the majority of the possible score range, and with little in the way of floor or ceiling effects. We note that HADS scores at baseline could (and did) go below the minimum of eight that applied at the time eligibility was assessed.

\section{Treatment Attendance and Retention Usual Care Condition}

CR exercise sessions were well attended, with a median of six sessions attended and $58.6 \%$ of patients attending at least six sessions.

\section{Intervention Condition}

Patients in Group-MCT had a high attendance rate at CR exercise sessions, with a median of six sessions attended and $52.2 \%$ of patients attending at least six sessions. Figure 3 summarizes the number of CR exercise sessions attended by group.
Overall, attendance at Group-MCT was high, with 13 patients (56.5\%) attending at least four out of six sessions of which 11 (47.8\%) attended all six. However, five patients $(21.7 \%)$ did not attend any sessions and five $(21.7 \%)$ attended only one or two.

\section{Four-Month Follow-Up}

At 4 -month follow-up $72.4 \%$ and $69.6 \%$ of control and intervention group participants, respectively, returned followup questionnaires.

One control group participant formally withdrew from the study (3.5\%), though this was immediately after randomization. Six control group patients did not return questionnaires at follow-up (20.7\%), and one questionnaire was lost in the post (3.5\%).

In comparison, four (17.4\%) MCT-arm participants formally withdrew from the study before 4-month follow-up. Reasons for withdrawal included no longer having enough time to commit to the study $(\mathrm{n}=1)$, and no longer being interested in the study $(\mathrm{n}=$ 3). However, as only two additional MCT-arm patients failed to return the 4-month follow-up the overall response was similar. One questionnaire was lost in the post $(4.4 \%)$.

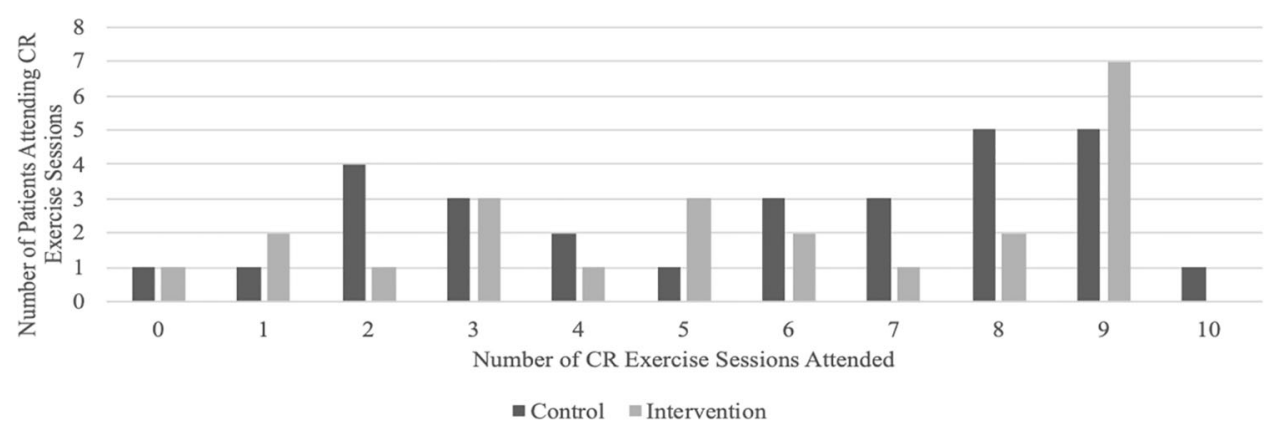

FIGURE 3 | Number of cardiac rehabilitation exercise sessions attended by group. 


\section{Group-MCT Adherence}

We examined therapists' level of adherence to the treatment protocol since the treatment was to be administered by nonmental health specialists with no experience of delivering psychological treatments. Therapists completed an adherence checklist at the end of each session to indicate which aspects of the session had been completed. Each site had two CR staff members trained to deliver MCT, one site had three staff members trained. Adherence to the protocol was high across all three sites, with an average adherence rating across sites of 98.2\%. Each site deviated from the protocol once; one site was unable to practice SpACE in session three as the session overran, while the remaining two sites both failed to review the previous session's homework. One site failed to review the homework in session five while the other site did not review the homework in session six.

\section{Eligibility Rates Before Baseline}

There was an unavoidable delay between patients having their initial CR assessment appointment and their subsequent study baseline assessment. This period was on average 7.5 days ( $\mathrm{SD}=5.95)$, but went as high as 31 days; however, baseline assessment was always completed prior to a patient's first cardiac rehabilitation session (i.e. first exercise class). HADS scores may change over this time, resulting in patients falling below the eligibility criteria of 8 points on the anxiety or depression subscale, and some may even spontaneously experience full clinical recovery. To assess the extent of these risks, we examined the change in HADS scores between initial assessment and study baseline. Five patients (10\%) made a clinically significant improvement (i.e. made a seven-point change on the HADS, calculated using (49), and two fell below the cutoff score of 8 points. One patient was classified as recovered (i.e. made a seven point change on the HADS and crossed the cutoff score).

\section{Sample Size Confirmation}

The Pathway RCT is a superiority trial comprising of an initial internal pilot followed by a main trial. The main trial was designed to detect an effect size of 0.4 on HADS total and to have $80 \%$ power not including the pilot sample. Under assumptions of $25 \%$ attrition, a correlation of 0.5 between baseline and follow-up outcome scores, mean therapy group size of 5.75, and intra-cluster correlation coefficient (ICC) of 0.05 , we estimated that a total recruitment sample of 230 patients was required for the main trial. Should merging with the internal pilot data prove feasible the final combined sample would be 280 , and the power $85 \%$.

We revised this estimate on the basis of the results of this internal pilot, which indicated an attrition rate of $35 \%$, over-time correlation of 0.5 (unchanged), mean group size of 3 and ICC of 0.05 (assumed). Since no substantial changes were made to the trial procedures or instruments following the pilot, with the consent of our steering committee and funders the decision was taken to merge the pilot data with the main trial. Considering the available time and resources, it was also decided to increase the recruitment target to 332 , to give the full study $90 \%$ power to detect the desired 0.4 effect size.

\section{Adverse Events}

Adverse events and serious adverse events were monitored for individuals in the intervention group. No adverse events were reported. For further details on the safety reporting protocol for the trial see (32).

\section{DISCUSSION}

Anxiety and depression are common in cardiac patients; however, current psychological interventions for cardiac patients are limited in efficacy, and as such novel psychological interventions are needed. The current study evaluates the acceptability of GroupMCT in cardiac patients and the feasibility of conducting a full randomized trial to compare Group-MCT plus treatment as usual to treatment as usual in this patient population.

Group-MCT was found to be both an acceptable and feasible treatment to deliver to cardiac patients with anxiety and depression. Recruitment and retention of participants to a psychological trial is often difficult (50); however, the current study successfully recruited to target. Recruitment rates were in line with previous studies evaluating psychological interventions within CR patients, whereby approximately 35\% of eligible patients agreed to take part in research (51). The HADS scores of participants in the study were also similar to those obtained in routine practice in the UK (52), which supports the generalizability of the study to clinical settings. Although the study did recruit to target there were challenges with recruitment, for example, some patients were not screened for eligibility due to limited CR staff or time to complete eligibility questionnaires. As such, future studies should consider providing additional support to CR staff during assessment clinics to assist with screening patients for eligibility. Reasons for not taking part in the trial included not having the time to commit to the study, returning to work, and not being interested in the study. Interestingly, one patient noted that despite scoring high on the HADS they did not feel as though they were anxious or depressed and therefore did not feel as though the research study was suitable for them.

Completion rates of CR were high at $75 \%$, which is in line with The National Audit of Cardiac Rehabilitation Annual report, which highlights that nationally in the UK $77 \%$ of patients complete CR (9). Completion of CR did not differ by group or by site, suggesting that the addition of Group-MCT does not impact on patient willingness to attend CR. Attendance at Group-MCT was moderate to good, with more than half of all patients attending at least four out of six sessions of Group-MCT, and most of these attending all six. We consider attendance at a minimum of four sessions to represent sufficient exposure to benefit appreciatively from the intervention. However, around one fifth of intervention patients did not attend any sessions, with a majority of these not providing a reason for nonattendance. Attendance at Group-MCT in the current study was slightly lower than some previous studies, but comparisons 
are difficult to interpret because not all other studies report attendance rates, and the studies are not within a cardiac sample.

Retention of participants in both trial groups was high. There was some evidence for a higher-though still small-number of participants randomized to Group-MCT formally withdrawing from the study, but this was balanced by a greater rate of return of follow-up questionnaires from this group. There appeared to be no indication that attending Group-MCT led to a reduction in attendance at CR sessions. It is important to note that reasons for withdrawal from the study may have been influenced by the format of delivery of the intervention. Some patients may not feel comfortable attending a group intervention and may have preferred a psychological intervention delivered on a one-toone basis.

The study had a high rate of return of follow-up questionnaires with over $70 \%$ returned. The study used a broad range of strategies to encourage returns including phone calls to check that patients had received their follow-up questionnaire packs, reminder phone calls asking patients to return them, and incentivizing return of questionnaires. In addition, we also provided patients with a range of options which included completing questionnaires over the phone and face-to-face with a research assistant. While a range of options were provided future studies should also consider offering electronic data collection, which may help to increase questionnaire return rates.

While the therapists reported a high level of adherence to the manual we were unable to assess the quality of therapy delivered throughout. This was due to limitations with audio recording therapy sessions. In future, trials should not only assess adherence to the manual but also quality of treatment delivery via an independent rater.

On the basis of the results reported here extension of the study to a full-scale trial was recommended. The results of the feasibility study were reported to the trial steering committee and the funder (NIHR), and the decision to extend recruitment to a full-scale RCT was supported.

\section{CONCLUSIONS}

The implementation of psychological treatments for anxiety and depression in physical health pathways presents several challenges. Existing treatments have limited efficacy and are not widely accessible. Furthermore, physical health care providers typically lack the knowledge and experience necessary to deliver complex psychological interventions. With these barriers in mind, we evaluated the feasibility and acceptability of running a trial of MCT in cardiac rehabilitation patients and delivered by nonmental health specialist staff. Our feasibility study was implemented as an internal pilot study, with the intention that if findings were favorable the study would be extended by continuing recruitment to a full-scale RCT.

The results suggest that a trial of MCT within the rehabilitation pathway is feasible. The addition of MCT to rehabilitation did not appear to have a negative impact on $\mathrm{CR}$ retention. Most participants completed our a-priori definition of a minimally effective dose of the intervention. Completion rates of measures were good suggesting they did not over-burden participants. The treatment approach also appeared feasible and acceptable to health providers as we observed high levels of therapist adherence to the protocol.

\section{DATA AVAILABILITY STATEMENT}

The datasets generated for this study are available on request to the corresponding author.

\section{ETHICS STATEMENT}

The studies involving human participants were reviewed and approved by National Research Ethics Service of the UK's National Health Service (ref 14/NW/0163). The patients/ participants provided their written informed consent to participate in this study.

\section{AUTHOR CONTRIBUTIONS}

AW, DR, PF, LD, AH, and PD were involved in study conception or design, and contributed to the first and subsequent drafts of the manuscript. DR and $\mathrm{CH}$ contributed to data analysis. LC drafted the manuscript and contributed to data analysis. $\mathrm{DR}, \mathrm{CH}$, AW, and LC contributed to interpretation of results. All agree to be accountable for all aspects of the work ensuring integrity and accuracy.

\section{FUNDING}

This study represents independent research funded by the National Institute for Health Research (NIHR) under its Programme Grants for Applied Research (PGfAR) Programme (Grant Reference Number RP-PG-1211-20011). The views expressed are those of the author(s) and not necessarily those of the NIHR or the Department of Health.

\section{ACKNOWLEDGMENTS}

The authors would like to thank the NIHR for their support and members of the PATHWAY team: Dr. Rebecca McPhillips, Mrs. Helen Morley, Dr. Hannah Gaffney, Ms. Rebecca Anderson, Dr. Cintia Faija, Dr. Kirsten McNicol, Ms. Gemma Shields, and Mrs. Wendy Clarke, who contributed towards participant recruitment and study organization. We would also like to thank the Trial Steering Committee: Prof. Kate Jolly, Prof. Gill Lancaster, Prof. Gerry Humphris, and Mr. Chris Houston. 


\section{REFERENCES}

1. British Heart Foundation (BHF). UK Fact Sheet. British Heart Foundation (2019). Available From: https://www.bhf.org.uk/what-we-do/our-research/ heart-statistics.

2. British Association for Cardiovascular Prevention and Rehabilitation (BACPR). BACPR Standards and Core Components, 3rd Edition. British Association of Cardiovascular Prevention and Rehabilitation (2017). Retrieved From: http://www.bacpr.com/resources/6A7_BACR_Standards_ and_Core_Components_2017.pdf.

3. National Institute for Health and Care Excellence (NICE). Chronic heart failure in adults: diagnosis and management. NICE guideline 106. National Institute for Health and Care Excellence (2018). Retrieved from: https://www. nice.org.uk/guidance/ng106.

4. National Health Service (NHS). The NHS Long Term Plan. National Health Service (2019). Available from: https://www.longtermplan.nhs.uk/wpcontent/uploads/2019/08/nhs-long-term-plan-version-1.2.pdf.

5. Anderson L, Thompson DR, Oldridge N, Zwisler AD, Rees K, Martin N, et al. Exercise-based cardiac rehabilitation for coronary heart disease. Cochrane Database Syst Rev (2016) 1:CD001800. doi: 10.1002/14651858. CD001800.pub3

6. Shields GE, Wells A, Doherty P, Heagerty A, Buck D, Davies LM. Costeffectiveness of cardiac rehabilitation: a systematic review. Heart (2018) 104:1403-10. doi: 10.1136/heartjnl-2017-312809

7. Dalal HM, Doherty P, Taylor RS. Cardiac rehabilitation. BMJ (2015) 351: h5000. doi: 10.1136/bmj.h5000

8. Anderson L, Taylor RS. Cardiac rehabilitation for people with heart disease: an overview of Cochrane systematic reviews. Cochrane Database Syst Rev (2014) 12:CD011273. doi: 10.1002/14651858.CD011273.pub2

9. National Audit of Cardiac Rehabilitation (NACR). Annual Report. York: British Heart Foundation, University of York (2018). Accessed From: https:// www.bhf.org.uk/informationsupport/publications/statistics/national-auditof-cardiac-rehabilitation-quality-and-outcomes-report-2018.

10. McGrady A, McGinnis R, Badenhop D, Bentle M, Muhammad R. Effects of Depression and Anxiety on Adherence to Cardiac Rehabilitation. J Cardiopulmonary Rehabil Prev (2009) 29(6):358-64. doi: 10.1097/ hcr.0b013e3181be7a8f

11. Frasure-Smith N, Lespérance F, Bentle M, Muhammad R. Depression and Anxiety as Predictors of 2-Year Cardiac Events in Patients With Stable Coronary Artery Disease. Arch Gen Psychiatry (2008) 65(1):62. doi: 10.1001/archgenpsychiatry.2007.4

12. Sherwood A. Relationship of Depression to Death or Hospitalization in Patients With Heart Failure. Arch Internal Med (2007) 167(4):367. doi: 10.1001/archinte.167.4.367

13. Richards SH, Anderson L, Jenkinson CE, Whalley B, Rees K, Davies P, et al. Psychological interventions for coronary heart disease. Cochrane Database Systemat Rev (2018) 4:CD002902. doi: 10.1002/14651858. CD002902.pub4

14. Dickens C, Cherrington A, Adeyemi I, Roughley K, Bower P, Garrett C, et al. Characteristics of Psychological Interventions That Improve Depression in People With Coronary Heart Disease: A Systematic Review and MetaRegression. Psychosomatic Med (2013) 75(2):211-21. doi: 10.1097/ PSY.0b013e31827ac009

15. Reavell J, Hopkinson M, Clarkesmith D, Lane DA. Effectiveness of Cognitive Behavioural Therapy for Depression and Anxiety in Patients with Cardiovascular Disease A Systematic Review and Meta-Analysis. Psychosomatic Med (2018) 80(8):742-53. doi: 10.1097/PSY.0000000 000000626

16. Wells A. Metacognitive therapy for anxiety and depression. New York: Guilford Press (2009).

17. Normann N, Morina N. The Efficacy of Metacognitive Therapy: A Systematic Review and Meta-Analysis. Front Psychol (2018) 9:2211. doi: 10.3389/ fpsyg.2018.02211

18. Wells A, Matthews G. Attention and emotion: A clinical perspective. Hove: Erlbaum (1994).

19. Wells A, Matthews G. Modelling Cognition in Emotional Disorder: The SREF MODEL. Behav Res Ther (1996) 34(11):881-8. doi: 10.1016/S0005-7967(96) 00050-2
20. Papageorgiou C, Carlile K, Thorgaard S, Waring H, Haslam J, Horne L, et al Group Cognitive-Behavior Therapy or Group Metacognitive Therapy for Obsessive-Compulsive Disorder? Benchmarking and Comparative Effectiveness in a Routine Clinical Service. Front Psychol (2018) 9:2551. doi: 10.3389/fpsyg.2018.02551

21. Rees CS, van Koesveld KE. An open trial of group metacognitive therapy for obsessive-compulsive disorder. J Behav Ther Exp Psychiatry (2008) 39(4):4518. doi: 10.1016/j.jbtep.2007.11.004

22. Haseth S, Solem S, Sørø GB, Bjørnstad E, Grøtte T, Fisher P. Group Metacognitive Therapy for Generalized Anxiety Disorder: A Pilot Feasibility Trial. Front Psychol (2019) 10:290. doi: 10.3389/fpsyg.2019.00290

23. McEvoy PM, Erceg-Hurn DM, Anderson RA, Campbell BNC, Swan A, Saulsman LM, et al. Group metacognitive therapy for repetitive negative thinking in primary an non-primary generalized anxiety disorder: An effectiveness trial. J Affect Disord (2015) 175(1):124-32. doi: 10.1016/ j.jad.2014.12.046

24. van der Heiden C, Melchior K, de Stigter E. The Effectiveness of Group Metacognitive Therapy for Generalised Anxiety Disorder: A Pilot Study. J Contemp Psychother (2013) 43:151-7. doi: 10.1007/s10879-013-9235-y

25. Papageorgiou C, Wells A. Group Metacognitive Therapy for Severe Antidepressant and CBT Resistant Depression: A Baseline-Controlled Trial. Cognit Ther Res (2015) 39:14-22. doi: 10.1007/s10608-014-9632-x

26. Dammen T, Papageorgiou C, Wells A. An open trial of group metacognitive therapy for depression in Norway. Nordic J Psychiatry (2015) 69(2):126-31. doi: $10.3109 / 08039488.2014 .936502$

27. Capobianco L, Reeves D, Morrison AP, Wells A. Group Metacognitive Therapy vs. Mindfulness Meditation Therapy in a Transdiagnostic Patient Sample: A Randomised Feasibility Trial. Psychiatry Res (2018) 250:554-61. doi: 10.1016/j.psychres.2017.11.045

28. Callesen P, Capobianco L, Heal C, Juul C, Find Nielsen S, Wells A. A Preliminary Evaluation of Transdiagnostic Group Metacognitive Therapy in a Mixed Psychological Disorder Sample. Front Psychol (2019) 10:1341. doi: 10.3389/fpsyg.2019.01341

29. Fisher PL, Byrne A, Fairburn L, Ullmer H, Abbey G, Salmon P. Brief Metacognitive Therapy for Emotional Distress in Adult Cancer Survivors. Front Psychol (2019). 10:162 doi: 10.3389/fpsyg.2019.00162

30. Fisher PL, Byrne A, Salmon P. Metacognitive therapy in adult survivors of cancer: a case series. Cogn Ther Res (2017) 41:891-901. doi: 10.1007/s10608017-9862-9

31. Fisher PL, McNicol K, Young B, Smith E, Salmon P. An open trial of metacognitive therapy for emotional distress in young adult survivors of cancer. J Adolesc Young Adult Oncol (2015) 4:64-9. doi: 10.1089/jayao. 2014.0046

32. Wells A, McNicol K, Reeves D, Salmon P, Davies L, Heagerty A, et al. Improving the effectiveness of psychological interventions for depression and anxiety in the cardiac rehabilitation pathway using group-based metacognitive therapy (PATHWAY Group MCT): study protocol for a randomised controlled trial. Trials (2018) 19:215. doi: 10.1186/s13063-018-2593-8

33. Zigmond AS, Snaith RP. The hospital anxiety and depression scale. Acta Psychiatr Scand (1983) 67(6):361-70. doi: 10.1111/j.1600-0447.1983. tb09716.x

34. Eldridge SM, Chan CL, Campbell MJ, Bond CM, Hopewell S, Thaban L, et al. COSNORT 2010 statement: extension to randomised pilot and feasibility trials. BMJ (2016) 355:i5239. doi: 10.1136/bmj.i5239

35. Hertzog MA. Considerations in determining sample size for pilot studies. Res Nurs Health (2008) 31(2):180-91. doi: 10.1002/nur.20247

36. Greenberger D, Padesky C. Mind Over Mood: A Cognitive Therapy Treatment Manual for Clients. New York: Guilford Press (1995).

37. Wells A. Group Metacognitive Therapy: Treatment Manual. Manchester, UK (2015).

38. Martin CR, Lewin RJP, Thompson DR. A confirmatory factor analysis of the Hospital Anxiety and Depression Scale in coronary care patients following acute myocardial infarction. Psychiatry Res (2003) 120(1):85-94. doi: 10.1016/ S0165-1781(03)00162-8

39. Roberts SB, Bonnici DM, Mackinnon AJ, Worcester MC. Psychometric evaluation of the Hospital Anxiety and Depression Scale (HADS) among female cardiac patients. Br J Health Psychol (2001) 6(4):373-83. doi: 10.1348/ 135910701169278 
40. Weiss DS, Marmar CR. The impact of event scale - revised. In: Wilson JP, Keane TM, editors. Assessing psychological trauma and PTSD. New York: Guilford Press (1997). p. 399-411.

41. Beck JG, DeMond GM, Read JP, Clapp JD, Coffey SF, Miller LM, et al. The impact of event scale-revised: psychometric properties in a sample of motor vehicle accident survivors. J Anxiety Disord (2008) 22(2):187-98. doi: 10.1016/ j.janxdis.2007.02.007

42. Creamer M, Bell R, Failla S. Psychometric properties of the impact of event scale - revised. Behav Res Ther (2003) 41:1489-96. doi: 10.1016/ j.brat.2003.07.010

43. Wells A, Cartwright-Hatton S. A short form of the metacognitions questionnaire: Properties of the MCQ-30. Behav Res Ther (2004) 42 (4):385-96. doi: 10.1016/S0005-7967(03)00147-5

44. Spada MM, Mohiyeddini C, Wells A. Measuring metacognitions associated with emotional distress: factor structure and predictive validity of the metacognitions questionnaire 30. Personal Individ Differ (2008) 45:238-42. doi: $10.1016 /$ j.paid.2008.04.005

45. Wells A. Cognitive Attentional Syndrome Scale 1 Revised (CAS-1R). Manchester: University of Manchester (2015).

46. Faija C, Reeves D, Heal C, Capobianco L, Anderson R, Wells A. Measuring the Cognitive Attentional Syndrome in Cardiac Patients with Anxiety and Depression Symptoms: Psychometric Properties of the CAS-1R. Front Psychol (2019) 10:2109. doi: 10.3389/fpsyg.2019.02109

47. EuroQol Group. EuroQol - a new facility for the measurement of healthrelated quality of life. Health Policy (1990) 16(3):199-208. doi: 10.1016/01688510(90)90421-9

48. Brooks R. EuroQol: The current state of play. Health Policy (1996) 37:53-72. doi: 10.1016/0168-8510(96)00822-6
49. Jacobson NS, Truax P. Clinical significance: a statistical approach to defining meaningful change in psychotherapy research. J Consult Clin Psychol (1991) 59(1):12-9. doi: 10.1037//0022-006x.59.1.12

50. Liu Y, Pencheon E, Hunter RM, Moncrieff J, Freemantle N. Recruitment and retention strategies in mental health trials- A systematic review. PloS One (2018) 3(8):e0203127. doi: 10.1371/journal.pone.0203127

51. Turner A, Hambridge J, Baker A, Bowman J, McElduff P. Randomised controlled trial of group cognitive behaviour therapy versus brief intervention for depression in cardiac patients. Austrialian New Zelands J Psychiatry (2012) 47(3):235-43. doi: 10.1177/0004867412460592

52. Sever S, Doherty P, Harrison AS, Golder S. To what extent is multi-morbidity associated with new onset depression in patients attending cardiac rehabilitation? BMC Cardiovasc Disord (2019) 19:256. doi: 10.1186/s12872-019-1245-6

Conflict of Interest: AW is the developer of metacognitive therapy and a codirector of the Metacognitive Therapy Institute.

The remaining authors declare that the research was conducted in the absence of any commercial or financial relationships that could be construed as a potential conflict of interest.

Copyright (c) 2020 Wells, Reeves, Heal, Fisher, Davies, Heagerty, Doherty and Capobianco. This is an open-access article distributed under the terms of the Creative Commons Attribution License (CC BY). The use, distribution or reproduction in other forums is permitted, provided the original author(s) and the copyright owner(s) are credited and that the original publication in this journal is cited, in accordance with accepted academic practice. No use, distribution or reproduction is permitted which does not comply with these terms. 